\title{
Efecto de una Pasta Dental Comercial Conteniendo Xilitol Sobre el Recuento de Streptococcus Mutans en Saliva de Gestantes: Ensayo Clínico Controlado Aleatorizado
}

\author{
Effect of a Commercial Toothpaste Containing Xylitol on the Count of Streptococcus \\ Mutans in Saliva of Pregnant Women: Randomized Controlled Clinical Trial
}

Roxana Patricia Escalante-Medina ${ }^{1,2}$; Angel Steven Asmat-Abanto ${ }^{3,4,5,6}$ \& Miguel Angel Ruiz-Barrueto Met, $^{7,8}$

ESCALANTE-MEDINA, R. P.; ASMAT-ABANTO, A. S. \& RUIZ-BARRUETO, M. A. Efecto de una pasta dental comercial conteniendo xilitol sobre el recuento de Streptococcus mutans en saliva de gestantes: ensayo clínico controlado aleatorizado. Int. J. Odontostomat., 13(3):316-320, 2019.

RESUMEN: El objetivo del presente trabajo fue determinar el efecto de una pasta dental comercial conteniendo xilitol sobre el recuento de Streptococcus mutans en saliva de gestantes. El presente fue un ensayo clínico, a doble ciego, que se realizó en el Centro de Salud "José Olaya" (Chiclayo Perú), en enero de 2017. Se trabajó con una población muestral de 50 gestantes en el segundo trimestre que cumplieron con los criterios establecidos, distribuyéndolas en dos grupos: 25 gestantes usaron pasta dental con $10 \%$ de xilitol y 25 gestantes usaron pasta dental sin xilitol. Se les tomó y procesó microbiológicamente una muestra de saliva antes del inicio del estudio y 14 días después del uso de las respectivas pastas. Se realizó el recuento de unidades formadoras de colonias (UFC) de Streptococcus mutans en saliva con una confiabilidad altamente significativa mediante el Coeficiente de Correlación Intraclase, calibración intra e interexaminador $(1,000$ y 0,999 , respectivamente). El análisis de los datos se realizó mediante la prueba U de Mann-Whitney, considerando un nivel de significancia del $5 \%$. No se encontró diferencia entre las gestantes que emplearon pasta dental con xilitol en comparación con las que utilizaron pasta sin xilitol $(p=0,062)$. Se concluyó que el efecto de la pasta dental comercial conteniendo xilitol es similar a una pasta sin xilitol sobre el recuento de Streptococcus mutans en saliva de gestantes.

PALABRAS CLAVE: Pasta dental, xilitol, Streptococcus mutans.

\section{INTRODUCCIÓN}

Durante el embarazo se producen muchos cambios fisiológicos. Algunos de estos también se manifiestan a nivel oral (Pentapati et al., 2013; Vamos et al., 2015; Kamate et al., 2017; Silva de Araujo Figueiredo et al., 2017), apreciándose un incremento en el número de microorganismos, lo que conlleva a un aumento de la placa dental y de la acidez salival y en consecuencia a una disminución del efecto buffer (Vergnes et al., 2012; Kamate et al.; Silva de Araujo
Figueiredo et al.). Esto, asociado a una modificación en la conducta alimentaria y en la higiene bucal, pueden promover el desarrollo de caries dental, lo que no sólo mermará la salud integral de la gestante, sino la del futuro bebé (Weber-Gasparoni et al., 2012; Vamos et al.; Kloetzel et al., 2017).

La caries dental es una enfermedad dinámica, multifactorial que causa la desmineralización y

\footnotetext{
${ }^{1}$ Cirujano Dentista. Maestra en Estomatología.

${ }^{2}$ Docente de la Escuela de Odontología de la Universidad Particular de Chiclayo, Chiclayo, Perú.

${ }^{3}$ Cirujano Dentista. Doctor en Estomatología.

${ }^{4}$ Docente de la Escuela de Postgrado de la Universidad Señor de Sipán, Chiclayo, Perú.

${ }^{5}$ Docente de la Escuela de Estomatología de la Universidad Privada Antenor Orrego, Trujillo, Perú.

${ }^{6}$ Docente. Universidad Señor de Sipán, Chiclayo, Perú.

${ }^{7}$ Biólogo Microbiólogo. Maestro en ciencias con mención en Microbiología Clínica.

${ }^{8}$ Docente Ordinario Auxiliar en Universidad César Vallejo, Piura, Perú.
} 
remineralización de los tejidos dentales duros (Pitts et al., 2017), es considerada la más frecuente de la cavidad oral (Lin et al., 2016). Asimismo, el género bacteriano Streptococcus constituye el principal grupo microbiano residente en el entorno dentario (Dhotre et al., 2016.) siendo Streptococcus mutans el agente más importante asociado al progreso de caries dental (Biswas \& Biswas, 2012; Ojeda-Garcés et al., 2013; Alamoudi et al., 2014; Dhotre et al.; Lin et al.) debido a su capacidad de utilizar la sacarosa para producir polisacáridos extracelulares adhesivos como los glucanos a través de la glucosiltransferasas (GTF) que promueven la formación de biopelículas y la capacidad de adherirse a las superficies dentarias (Jung et al., 2016; Chu et al., 2016; Nilsson et al., 2016).

Para mantener su presencia dominante y generar caries dental, S.mutans, (Biswas \& Biswas; Ojeda-Garcés et al.; Alamoudi et al.) puede reducir drástica y rápidamente el $\mathrm{pH}$ de su entorno. La exposición del esmalte dental a estos niveles de acidez conllevan a su desmineralización.

Esta bacteria también tiene la capacidad de secretar péptidos antimicrobianos (mutacinas) para suprimir el crecimiento de otras especies competidoras y favorecer así su proliferación (Biswas \& Biswas; Nilsson et al.).

Las estrategias actuales destinadas a prevenir la caries están dirigidas hacia el control de los microorganismos específicos y la disminución de la ingesta de azúcares fermentables principalmente la sacararosa. (Alamoudi et al.) El uso de la terapia de sustitución de edulcorantes como la sacarosa, por polialcoholes (Milgrom et al., 2012), y de hábitos alimentarios perjudiciales parecen tener un efecto positivo sobre los niveles de caries (Alamoudi et al.). El xilitol es un polialcohol de azúcar o alcohol polihídrico de azúcar de 5 carbonos (Söderling et al., 2011; Nayak et al., 2014).

Algunos estudios mostraron la capacidad de xilitol para disminuir el crecimiento de $S$. mutans y la producción de ácidos por bacterias cariogénicas (Silva et al., 2009). Asimismo, ayuda a reducir la formación de placa y aumenta el flujo salival, ayudando en la reparación del esmalte dañado, reduciendo el desarrollo de la caries dental.

El propósito del presente estudio fue determinar el efecto de una pasta dental comercial conteniendo xilitol sobre el recuento de Streptococcus mutans en saliva de gestantes.

\section{MATERIAL Y MÉTODO}

Cálculo de la muestra: La presente investigación fue de tipo experimental y correspondió a un ensayo clínico, a doble ciego, que se realizó en el Centro de Salud "José Olaya" en enero 2017 (Chiclayo,Perú). El tamaño de muestra por grupo fue de 20 gestantes, para determinarla se usó la fórmula para comparación de promedios, usando los parámetros siguientes: $\mathrm{Za}=0,010$ (Alfa, máximo error tipo I), Z b=0,050 (Beta, máximo error tipo II),s12=2,647(varianza del grupo con xilitol, obtenido de estudio piloto), s22=6,567(varianza del grupo sin xilitol, obtenido de estudio piloto). Al ser el tamaño muestral el número mínimo, se decidió evaluar 25 gestantes por grupo debido al riesgo de deserción en estudios de seguimiento.

Previamente al cálculo de la muestra, se realizó un estudio piloto, donde se evaluó la confiabilidad del método de recuento de unidades formadoras de colonias (UFC) en 20 placas Petri. Se hizo la calibración intra e interexaminador, mediante el Coeficiente de Correlación Intraclase, apreciándose una confiabilidad altamente significativa $(1,000$ y 0,999 respectivamente).

Criterios de selección de la muestra: Se incluyeron en el estudio, gestantes en el segundo trimestre sin enfermedades sistémicas, con caries dental y mayores de 18 años que acudieron al consultorio externo de obstetricia. Se excluyeron a las gestantes con prótesis dental (fija y/o removible), que se encontraron bajo tratamiento ortodóntico, que usaron regularmente productos con xilitol, que estén consumiendo antibióticos o hayan recibido tratamiento con antibióticos a largo plazo u otros medicamentos que afecten la microbiota oral, y a las que no aceptaron participar del estudio. Se eliminaron gestantes a las que no se les pudo realizar el segundo control y que presentaron complicaciones en su embarazo durante el estudio.

Para su ejecución, se contó con la aprobación de la Comisión de Investigación de la Escuela de Postgrado de la Universidad Señor de Sipán con Resolución N0067-2017/EPUSS-USS, considerándose los principios éticos de la Declaración de Helsinki y de la Ley General de Salud del Perú (Ley Nº 26842).

Previo al procedimiento se explicó a la gestante la importancia de la investigación y, si aceptó participar, se le solicitó la lectura y firma del consentimiento informado. 
Se registraron los datos personales de las participantes, distribuyéndolas aleatoriamente a los siguientes grupos:Grupo A: Uso pasta dental Xerox® conteniendo $10 \%$ de xilitol y Grupo B: Uso pasta dental Colgate Triple Acción ${ }^{\circledR}$

Toma de muestra inicial de saliva: Se tomó una muestra de saliva a las gestantes, en ayunas( a horas tempranas de la mañana) y sin cepillarse los dientes, indicándoles acumular la saliva por 2 minutos y escupir $(1,5 \mathrm{ml})$ en el tubo Eppendorff estéril. A cada gestante se le entregó el mismo tipo de cepillo y las pasta dentales según grupo de estudio en empaques iguales enumeradas del 1 al 50, con el apoyo de un colaborador ajeno al estudio.

Capacitación en técnica de cepillado: Posteriormente se les instruyó sobre la técnica de cepillado Bass modificada. Las gestantes se cepillaron con la pasta dental asignada después del desayuno, del almuerzo e inmediatamente antes de dormir, siguiendo las siguientes instrucciones:

1.Utilizar aproximadamente $1,5 \mathrm{~cm}$ de pasta dental (equivalente a $1 \mathrm{~g}$ ) en un cepillo de dientes húmedo (proporcionado por la investigadora). 2. Distribuir la pasta de dientes de manera uniforme en la arcada superior e inferior. 3. Cepillarse durante 2 minutos. 4. Utilizar un sorbo de agua junto con la pasta dental que queda en la boca y enjuagar durante 30 segundos antes de escupir. 5. Evitar comer y beber durante 2 horas posterior al cepillado. Se mantuvo contacto telefónico con la paciente, para monitorizar el cumplimiento de las indicaciones, hasta el día de control (14 días después de la toma de muestra inicial).

Procesamiento microbiológico de la muestra de saliva: Después de recolectadas las muestras de saliva, éstas se colocaron en un cooler con refrigerantes y fueron transportadas al laboratorio de Microbiología de la Universidad Señor de Sipán en un tiempo no mayor a 2 horas a fin de que la carga microbiana inicial no se vea alterada. Inmediatamente después de llegada las muestras de saliva al laboratorio, se reali- zó una dilución seriada de cada muestra en agua destilada estéril. La homogenización de las diluciones se realizó en un equipo Vórtex.

Con ayuda de una micropipeta se tomaron 100 $\mu \mathrm{l}$ de la sétima dilución de la muestra saliva y se depositó sobre la superficie de placas de petri conteniendo Agar TSY20 e inmediatamente después se procedió a realizar la siembra en dispersión con un asa de drigalsky. Las placas sembradas fueron incubadas a $36,5^{\circ} \mathrm{C}$ durante 24 horas en microaerofilia. Se sembró una cepa control de S. mutans ATCC 25175 para orientar el recuento y la identificación de la bacteria mediante pruebas bioquímicas.

Después de las 24 horas de incubación, las placas fueron retiradas de la incubadora para realizar el recuento de UFC/mL de $S$. mutans presente en las muestras de saliva de las gestantes. El recuento se hizo con un contador de colonias Boeco CC1. Por el tipo de distribución estadística que presentaron los resultados, el efecto de la pasta conteniendo xilitol fue analizada empleando la prueba $U$ de Mann-Whitney, considerando un nivel de significancia del $5 \%$.

\section{RESULTADOS}

Luego de aplicar los criterios de eliminación. La Muestra quedó reducida a 45 gestantes, cuyas edades oscilaron entre 25 y 35 años de edad (Media=30,6). 23 gestantes recibieron una pasta dental comercial conteniendo xilitol y 22 una pasta dental sin xilitol.

No se encontró diferencia entre las pastas con respecto a UFC, entre las gestantes que emplearon pasta dental con xilitol y las que utilizaron pasta sin xilitol. $(p=0,062)$, como se muestra en la Tabla I.

El uso de pasta dental comercial conteniendo xilitol permitió la disminución en el recuento de Streptococcus mutans en saliva de gestantes $(p=0,001)$, como se muestra en la Tabla II.

Tabla I. Efecto de una pasta dental con xilitol sobre el recuento de Streptococcus mutans en saliva de gestantes.

\begin{tabular}{cccccccc}
\hline Grupo & $\mathrm{n}$ & Media & \multicolumn{2}{c}{ I.C. } & Me & DE & $\mathrm{p}^{*}$ \\
\cline { 4 - 5 } & & & LI & LS & & & \\
\hline Con Xilitol & 23 & 3,830 & 2,019 & 5,642 & 3,000 & 4,190 & 0,062 \\
Sin Xilitol & 22 & 5,955 & 3,009 & 8,900 & 4,000 & 6,644 & \\
\hline
\end{tabular}

*U de Mann-Whitney; DE, desviación estándar; Me, mediana; I.C., intervalo de confianza al 95 $\%$; LI, límite inferior; LS, límite superior. 
ESCALANTE-MEDINA, R. P.; ASMAT-ABANTO, A. S. \& RUIZ-BARRUETO, M. A. Efecto de una pasta dental comercial conteniendo xilitol sobre el recuento de Streptococcus mutans en saliva de gestantes: ensayo clínico controlado aleatorizado. Int. J. Odontostomat., 13(3):316-320, 2019.

Tabla II. Eficacia de una pasta dental con xilitol para disminuir el recuento de Streptococcus mutans en saliva de gestantes.

\begin{tabular}{lccccccc}
\hline Variable & \multirow{2}{*}{$\mathrm{n}$} & Media & \multicolumn{2}{c}{$\mathrm{I}, \mathrm{C}}$, & Me & DE & \multirow{2}{*}{$\mathrm{p}^{*}$} \\
\cline { 5 - 6 } & & & $\mathrm{LI}$ & $\mathrm{LS}$ & & & \\
\hline UFC Antes & 23 & 12,000 & 6,169 & 17,831 & 6,000 & 13,484 & 0,001 \\
UFC Después & 23 & 3,830 & 2,019 & 5,642 & 3,000 & 4,190 & \\
\hline
\end{tabular}

Tabla III. Eficacia de una pasta dental sin xilitol para disminuir el recuento de Streptococcus mutans en saliva de gestantes.

\begin{tabular}{|c|c|c|c|c|c|c|c|}
\hline \multirow[t]{2}{*}{ Variable } & \multirow[t]{2}{*}{$\mathrm{n}$} & \multirow[t]{2}{*}{ Media } & \multicolumn{2}{|c|}{$\mathrm{I}, \mathrm{C}$} & \multirow[t]{2}{*}{$\mathrm{Me}$} & \multirow[t]{2}{*}{ DE } & \multirow[t]{2}{*}{$\mathrm{p}^{*}$} \\
\hline & & & $\mathrm{LI}$ & LS & & & \\
\hline UFC Antes & 22 & 14,409 & 6,753 & 22,065 & 5,000 & 17,267 & 0,005 \\
\hline UFC Después & 22 & 5,955 & 3,009 & 8,900 & 4,000 & 6,644 & \\
\hline
\end{tabular}

El uso de pasta dental sin xilitol fue eficaz para disminuir el recuento de Streptococcus mutans en saliva de gestantes $(p=0,005)$, como se muestra en la Tabla III.

\section{DISCUSIÓN}

La salud bucal es una parte importante de la salud pública, siendo trascendental durante la gestación (Bahria et al., 2015), puesto que en este estado existen cambios en la cavidad oral, los cuales asociados a conductas alimentarias con predilección de carbohidratos y deficiente higiene bucal permiten la formación de caries dental, repercutiendo no sólo en la salud de la gestante sino también en la de sus futuros bebés (Weber-Gasparoni et al.).

Los resultados obtenidos en el presente estudio no concuerdan con la hipótesis planteada. Se observó que no existe diferencia entre el uso de la pasta dental comercial conteniendo xilitol y sin xilitol, tal vez porque ambos grupos se lograron sensibilizar con la información brindada sobre la importancia de mantener la salud bucal y la transmisión de microorganismos de ellas a sus futuros bebés, de esta manera, cumplieron con las técnicas de higiene adecuada. Los resultados coinciden con el estudio de Surdacka \& Stopa (2005), quienes trabajaron en estudiantes de 21 a 25 años.

Sin embargo, los resultados encontrados discrepan con el estudio de Nakai et al. (2010) y Jannesson et al. (2002). En el caso de Nakai et al., 2010 quienes usaron goma de mascar con xilitol, concluyeron que los niños del grupo control adquirieron
S. mutans a una edad más temprana, que los niños del grupo xilitol. Probablemente en este estudio hubo diferencia porque se trabajó con un grupo que no recibió ninguna clase de goma de mascar, pudiendo haber influenciado la estimulación del flujo salival relacionada al uso de la goma de mascar con xilitol. El flujo salival está relacionado con la propiedad de la saliva en diluir los substratos bacterianos y azúcares ingeridos (Buzalaf et al., 2012). En el caso de Janennson et al , se trabajó con estudiantes de 25 años en promedio, donde concluyeron que la adición de $10 \%$ de xilitol a una pasta que contiene triclosan reduce el número de $S$. mutans en saliva. Esto posiblemente se haya debido a que la pasta dental utilizada, no contenía sólo xilitol, sino tambien triclosán.

Una limitación de esta investigación posiblemente sería el corto tiempo de uso de la pasta con xilitol. Se evaluó por 14 días, para evitar la probable deserción de las unidades de análisis. Otra limitación podría ser que la técnica de cepillado se enseñó sólo una vez y no se supervisó si cada vez que se cepillaban lo hacían siguiendo las indicaciones de dicha técnica. Sin embargo, esto se maneja dentro del error aleatorio que presentan todos los estudios similares.

\section{CONCLUSIONES}

Se concluye que el efecto de la pasta dental comercial conteniendo xilitol es similar a una pasta sin xilitol sobre el recuento de Streptococcus mutans en saliva de gestantes. Las pastas dentales con y $\sin x$ xilitol son eficaces para disminuir el recuento de Streptococcus mutans en saliva de gestantes. 
ESCALANTE-MEDINA, R. P.; ASMAT-ABANTO, A. S. \& RUIZ-BARRUETO, M. A. Effect of a commercial toothpaste containing xylitol on the count of Streptococcus mutans in saliva of pregnant women: randomized controlled clinical trial. Int. J. Odontostomat., 13(3):316-320, 2019.

ABSTRACT: The objective of the present study was to determine the effect of a commercial toothpaste containing xylitol on the counts of Streptococcus mutans in saliva of pregnant women. The present was a double-blind clinical trial performed at the "José Olaya" health Centre (Chiclayo Peru) in January 2017. We worked with a sample population of 50 pregnant in the second trimester that met the established criteria, distributing in two groups: 25 pregnant women used toothpaste with $10 \%$ xylitol and 25 pregnant used toothpaste without xylitol. They were taken and microbiologically processed a sample of saliva before the start of the study and 14 days after the use of the respective toothpastes. The Colony-forming units (CFU) of Streptococcus mutans in saliva were counted with a highly significant reliability through the intraclass correlation coefficient, Intra-and Interexaminer calibration ( 1.000 and 0.999 , respectively). Data analysis was performed using the Mann-Whitney $U$ test, considering a $5 \%$ significance level. No difference was found among the pregnant women who used xylitol toothpaste compared to those who used toothpaste without $x y$ litol $(p=0,062)$. It was concluded that the effect of xylitol containing commercial toothpaste is similar to a toothpaste without xylitol on the count of Streptococcus mutans in the saliva of pregnant women.

KEY WORDS: toothpaste, xylitol, streptococcus mutans.

\section{REFERENCIAS BIBLIOGRÁFICAS}

Alamoudi, N. M.; Hanno, A. G.; Almushayt, A. S.; Masoud, M. I.; El Ashiry, E. A. \& El Derwi, D. A. Early prevention of childhood caries with maternal xylitol consumption. Saudi Med. J., 35(6):592-7, 2014.

Bahria, N.; Tohidinik, H. R.; Bahrif, N.; lliati, H. R.; Moshki, M. \& Darabi, F. Educational intervention to improve oral health beliefs and behaviors during pregnancy: a randomized-controlled trial. J. Egypt. Public Health Assoc., 90(2):41-5, 2015.

Biswas, S. \& Biswas, I. Complete genome sequence of Streptococcus mutans GS-5, a serotype c strain. J. Bacteriol., 194(17):4787-8, 2012.

Buzalaf, M. A.; Hannas, A. R. \& Kato, M. T. Saliva and dental erosion. J. Appl. Oral Sci., 20(5):493-502, 2012.

Chu, J.; Zhang, T. \& He, K. Cariogenicity features of Streptococcus mutans in presence of rubusoside. B. M. C. Oral Health, 16(1):54, 2016.

Dhotre, S.; Suryawanshi, N.; Nagoba, B. \& Selkar, S. Rare and unusual isolates of viridans streptococci from the human oral cavity. Indian J. Pathol. Microbiol., 59(1):47-9, 2016.

Jannesson, L.; Renvert, S.; Kjellsdotter, P.; Gaffar, A.; Nabi, N. \& Birkhed, D. Effect of a triclosan-containing toothpaste supplemented with $10 \%$ xylitol on mutans streptococci in saliva and dental plaque. A 6-month clinical study. Caries Res., 36(1):36-9, 2002.

Jung, J. E.; Cai, J. N.; Cho, S. D.; Song, K. Y. \& Jeon, J. G. Influence of fluoride on the bacterial composition of a dual-species biofilm composed of Streptococcus mutans and Streptococcus oralis. Biofouling, 32(9):1079-87, 2016.
Kamate, W. I.; Vibhute, N. A. \& Baad, R. K. Estimation of DMFT, salivary Streptococcus mutans count, flow rate, ph, and salivary total calcium content in pregnant and non-pregnant women: a prospective study. J. Clin. Diagn. Res., 11(4):ZC147-51, 2017.

Kloetzel, M. K.; Huebner, C. E. \& Milgrom, P. Referrals for dental care during pregnancy. J. Midwifery Womens Health, 56(2):110-7, 2011.

Lin, H. K.; Fang, C. E.; Huang, M. S.; Cheng, H. C.; Huang, T. W.; Chang, H. T. \& Tam, K. W. Effect of maternal use of chewing gums containing xylitol on transmission of mutans streptococci in children: a metaanalysis of randomized controlled trials. Int. J. Paediatr. Dent., 26(1):35-44, 2016

Milgrom, P.; Söderling, E. M.; Nelson, S.; Chi, D. L. \& Nakai, Y. Clinical evidence for polyol efficacy. Adv. Dent. Res., 24(2):112-6, 2012.

Nayak, P. A.; Nayak, U. A. \& Khandelwal, V. The effect of xylitol on dental caries and oral flora. Clin. Cosmet. Investig. Dent., 6:89-94, 2014.

Nilsson, M.; Rybtke, M.; Givskov, M.; Høiby, N.; Twetman, S. \& TolkerNielsen, T. The dlt genes play a role in antimicrobial tolerance of Streptococcus mutans biofilms. Int. J. Antimicrob. Agents, 48(3):298304, 2016.

Ojeda-Garcés, J. C.; Oviedo-García, E. \& Salas, L. A. Streptococcus mutans y caries dental. CES Odontol., 26(1):44-56, 2013.

Pentapati, K. C.; Acharya, S.; Bhat, M.; Rao, S. K. \& Singh, S. Knowledge of dental decay and associated factors among pregnant women: a study from rural India. Oral Health Prev. Dent., 11(2):161-8, 2013.

Pitts, N. B.; Zero, D. T.; Marsh, P. D.; Ekstrand, K.; Weintraub, J. A.; Ramos-Gomez, F.; Ramos-Gomez, F.; Tagami, J.; Twetman, S.; Tsakos, G. \& Ismail, A. Dental caries. Nat. Rev. Dis. Primers, 3:17030, 2017.

Silva de Araujo Figueiredo, C.; Gonçalves Carvalho Rosalem, C.; Costa Cantanhede, A. L.; Abreu Fonseca Thomaz, É. B. \& Fontoura Nogueira da Cruz, M. C. Systemic alterations and their oral manifestations in pregnant women. J. Obstet. Gynaecol. Res., 43(1):16-22, 2017.

Silva, T. C.; Pereira, A. G. F.; Machado, M. A. A. M. \& Buzalaf, M. A. R. The use of xylitol as a strategy for prevention of dental caries. Rev. Odontol. Ciênc., 24(2):205-12, 2009.

Söderling, E.; Hirvonen, A.; Karjalainen, S.; Fontana, M.; Catt, D. \& Seppä, $\mathrm{L}$. The effect of xylitol on the composition of the oral flora: a pilot study. Eur. J. Dent., 5(1):24-31, 2011.

Surdacka, A. \& Stopa, J. The effect of xylitol toothpaste on the oral cavity environment. J. Prev. Med., 13(1-2):98-107, 2005.

Vamos, C. A.; Thompson, E. L.; Avendano, M.; Daley, E. M.; Quinonez, R. B. \& Boggess, K. Oral health promotion interventions during pregnancy: a systematic review. Community Dent. Oral Epidemiol., 43(5):385-96, 2015.

Vergnes, J. N.; Kaminski, M.; Lelong, N.; Musset, A. M.; Sixou, M.; Nabet, C. \& EPIPAP group. Frequency and risk indicators of tooth decay among pregnant women in France: a cross-sectional analysis. PLOS One, 7(5):e33296, 2012.

Weber-Gasparoni, K.; Goebel, B. M.; Drake, D. R.; Kramer, K. W.; Warren, J. J.; Reeve, J. \& Dawson, D. V. Factors associated with mutans streptococci among young WIC-enrolled children. J. Public Health Dent., 72(4):269-78, 2012.

Dirección para correspondencia:

Roxana Patricia Escalante Medina

Escuela de Odontología

Universidad Particular de Chiclayo

Chiclayo - PERÚ

Email: patriciaescalante_medina@hotmail.com

Recibido: 06-12-2018

Aceptado: 08-04-2019 